\title{
Image segmentation by geodesic voting. Application to the extraction of tree structures from confocal microscope images
}

\author{
Youssef Rouchdy and Laurent D. Cohen \\ CEREMADE, UMR7534 CNRS, Université Paris Dauphine \\ rouchdy@ceremade.dauphine,cohen@ceremade.dauphine.fr
}

\begin{abstract}
This study presents a new method to segment thin tree structures, such as extensions of microglia and cardiac or cerebral blood vessels. The Fast Marching method allows the segmentation of tree structures from a single point chosen by the user when a priori information is available about the length of the tree [4]. In our case, no a priori information about the length of the tree structure to extract is available. We propose here to compute geodesics from a set of points dispersed in the image. The targeted structure corresponds to image points with a high geodesic density. To compute the geodesic density we propose two methods. The first method defines the geodesic density of pixels in the image as the number of geodesics that cross this pixel. The second method consists in solving the transport equation with a velocity computed from the gradient of the distance map. In this method, the geodesic density is computed by integrating in short time the solution of the transport equation. To our knowledge this is the first time that geodesic voting is introduced. Numerical results from confocal microscope images are presented and show the interest of our approach.
\end{abstract}

\section{Introduction}

We are interested in the analysis of biological images acquired with a confocal microscope. The data are composed of a set of $3 \mathrm{D}+\mathrm{T}$ image sequences. For each time point, a series of 23 images perpendicular to the $z$ axis was acquired, thereby covering the three dimension of the cell. The aim is firstly, to segment the microglia extensions in 3D and secondly, to track it in time. The main difficulties with this data are: large deformations of the microglia extensions, which correspond to the tree structure, in time; presence of small features belonging to other cells and noise; the data is anisotropic: high resolution in the plane of the slice and lower resolution in the perpendicular direction. Hence, a simple use of the image intensities is not sufficient to extract directly the tree structure. Malladi et al. [8] used the Level Set methods to extract information from MRI data (which present approximately the same difficulties as the confocal microscope images). The Fast Marching method, introduced by Sethian in [12], and adapted by Cohen et al. [4] to extract tree structures, demands less computation time than the Level Set method and works with only one point chosen by the user on the tree. However, this method depends on a priori information about the target. In our case no a priori information about the tree structure is available. Here, we present an original method to extract tree structures without using any a priori information. The method is generic, it can be used to extract any type of tree structure in 2D as well as 3D. The method consists of extracting geodesics from several points in the image, from these points a geodesic density can be defined on the image with a high value of the density for the tree structure and a lower value outside of this. The segmentation result coresponds to pixels with a high density value. Figure 1 shows the principle of segmentation by geodesic voting on a simple synthetic image representing a segment. Right panel of (figure 1) shows the geodesic paths (black lines) which are superposed on the distance map associated to the synthetic image, the method to compute a distance map will be presented in the next section, left panel (figure1) represents the geodesic density, methods to compute this will be presented in the section 3 .

\section{Background}

\subsection{Minimal paths}

The minimal path theory for the extraction of contours from the image was inspired by the principle of Fermat: the light trajectory minimizes the optical dis- 


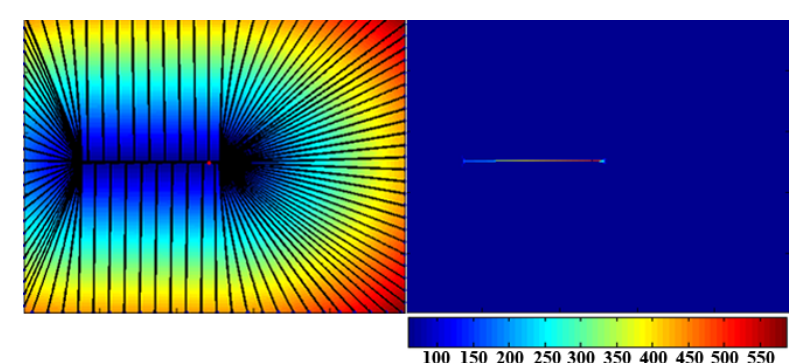

Figure 1. Geodesic voting method.

tance between $x_{0}=y(0)$ and $x=y(t)$, e.g. it gives the curve $y$ that minimizes the distance

$$
\tau\left(x_{0}, x\right)=\int_{0}^{t} \frac{\mathrm{d} s}{c(y(s))}
$$

where propagation speed $c$ is a function depending on the medium of the propagation. In homogenous media the function $c$ is a constant, the trajectories correspond to lines. In a medium with two regions, the function $c$ takes two values: $c_{1}$ in the first region and $c_{2}$ in the second region. The trajectory, in this case, corresponds usually to two joint segments, each segment belonging to one region. We are interested here in the case of a medium with a continuous velocity $c$. In the context of image segmentation Cohen and Kimmel proposed, in [5], a deformable model based on the optical distance (1). The model is formulated as a calculus of variation problem :

$$
\operatorname{Min} \int_{0}^{t}(w+P(y(s))) \mathrm{d} s,
$$

the minimum is considered in $\left\{y:[0, t] \longrightarrow \mathbb{R}^{2}\right.$ : $\left.y(0)=x_{0}, \quad y(t)=x\right\}$. The constant $w$ imposes regularity on the curve. $P>0$ is a potential computed from the image, it takes lower values near the edges or the features. For instance $P=I, \quad P=g(\|\nabla I\|)$, where $I$ is the image and $g$ is a decreasing function. To compute the solution associated to the source $x_{0}$ of this problem, we consider a Hamiltonian approach: Find the travel time $\mathrm{U}$ that solves the eikonal equation

$$
\|\nabla \mathrm{U}(x)\|=w+P(x) \quad x \in \Omega
$$

The ray $y$ is subsequently computed by backpropagation from $x$ by solving the ODE

$$
y^{\prime}(s)=-\nabla \mathrm{U}(y) .
$$

The only stable schemes that solve the eikonal equation compute a viscosity solution [6]. The first work that uses the viscosity solution is from Vidale [14]. Based on this work Fatemi et al. [7] proposed the first numerical scheme to solve the eikonal equation. To solve eikonal equation through iterations [11], at least $O\left(m n^{2}\right)$ are needed, where $n$ is the total number of grid points and $m$ is the number of iterations that permit an estimation of the solution. In the next section, an algorithm with the complexity $O(n \log (n))$ introduced in [12] is presented to solve this problem.

\subsection{Fast Marching method}

The idea behind the Fast Marching algorithm is to propagate the wave in only one direction, starting with the smaller values of the action map $U$ and progressing to the larger values using the upwind property of the scheme. Therefore, the Fast Marching method permits only one pass on the image starting from the sources in the downwind direction. Here, the principle of the Fast Marching method is given, for details see $[12,13]$. The grid points are partitioned into three dynamic sets: trial points, alive points and far points. The trial points correspond to a dynamic boundary that separates far points and alive points. At each step, the trial point with the minimum value of the action map $U$ is moved to the set of alive points, which are the grid points for which a value $\mathrm{U}$ has been computed. The values of alive points do not change. To reduce the computing time, the trial points are stocked in a data structure referred to as minheap (the construction of this data structure is described in $[12,13,3])$. The complexity to change the value of one element of the min-heap is $O(n)$. Hence, the total work for Fast Marching is $O(n \log (n))$. The Dijkstra algorithm, which is also used to find a minimal path, has the same complexity as the Fast Marching algorithm. However, the Dijkstra algorithm gives a linear approximation and there is no convergence result contrary to the Fast Marching algorithm, which converges toward the unique viscosity solution.

\section{Segmentation by geodesic voting of tree structures}

With the Fast Marching method we can extract the minimal path between two points. Here, the aim is to extract a tree with just one point chosen by the user. When a priori information is available about the length of the contour that one wants to extract, Cohen and al. proposed in [4] a method to extract a tree structure from one point selected by the user. In the following sections, a method is proposed for the segmentation of tree structures from only one given point without having any $a$ priori information about the tree to extract. The method 


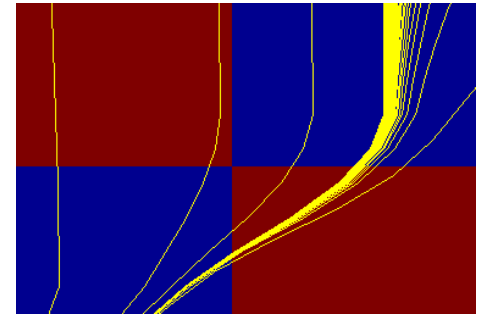

Figure 2. Density of geodesic paths. Yellow lines represent geodesic paths crossing four pixels, the pixels are represented by the blue and brown blocks. The geodesic density of the pixels in the image correspond to the number of trajectories crossing the given pixel.

uses a new concept for the image segmentation. This method consists in computing the geodesic density from several geodesics extracted from the image.

\subsection{Voting by characteristics}

The method consists in computing a characteristics curve from image points. The tree structure corresponds to the points with high geodesic density. First we choose the root of the tree structure and we propagate a front in the whole image with the Fast Marching method. Then, the geodesic paths from the border of the image and from points dispersed in the image, denoted by $\left\{x_{k}\right\}_{k=1}^{N}$, is extracted by solving the ODEs:

$$
\frac{\partial y_{k}}{\partial s}=-\nabla \mathrm{U}(y(s)), \quad y_{k}(0)=x_{k}, k=1, \cdot, N
$$

We define the voting score or the geodesic density at each point $p$ of the image by

$$
\mu(p)=\sum_{k=1}^{N} \delta_{p}\left(y_{k}\right)
$$

the function $\delta_{p}(y)$ returns 1 if the path $y$ crosses the pixel $p$, else 0 , see figure 2 .

\subsection{Voting with a transport equation}

The trajectories $y_{k}$ computed from (5) are called characteristics for the conservation equation

$$
\left.u_{t}+\operatorname{div}(v u)=0, \quad(t, x) \in\right] 0, T[\times \Omega,
$$

where $v=-\nabla \mathrm{U}$ denotes the velocity field computed from the distance map U. Due to the conservation of the information transported by the equation (7) toward the source point, we can define a geodesic density as the integral of the solution of the transport equation (7) in the short time $T$

$$
\mu(x)=\int_{0}^{T} u(t, x) \mathrm{d} t .
$$

By integration of the transport equation (7) with respect to the time $t$, we get

$$
\operatorname{div}(v \mu)=u(0, x)-u(T, x), \quad x \in \Omega
$$

The partial differential equation (9) is not elliptic, so it is more convenient to compute the geodesic density by the relation (8) after solving the transport equation (7).

\subsection{Schemes for the transport equation}

Medical images contain noise due to the imperfection of the acquisition system and the motion of the target. Furthermore the velocity field computed from the image is irregular. We propose therefore a method to solve the transport equation (7) based on the space-time integrated least square (STILS) method introduced in [9]. Recently, existence and unicity results for the minimizer of this method have been proved in [2] for an irregular velocity field. Numerical discretization is given for example in [1] when the divergence of the vector field $v$ is null. Here, we extend this method to the case that divergence of the velocity is non null and irregular.

Let the application $S$ defined by

$$
S: u \longrightarrow S(u)=u_{t}+\operatorname{div}(v u) .
$$

Instead of solving the transport equation (7), STILS method consists in minimizing the functional

$$
J(u)=\frac{1}{2} \int_{\Omega \times[0, T]} S(u)^{2} \mathrm{~d} x \mathrm{~d} t
$$

In the following, some definitions to construct a scheme to approximate the minimizer of the functional $J$ are given. We define a bilinear form $\mathcal{B}$ by

$$
\mathcal{B}(u, w)=\int_{\Omega \times[0, T]} S(u) S(w) \mathrm{d} x \mathrm{~d} t .
$$

Let $M$ be a positif number, $\left\{\psi_{1}(x), \cdots, \psi_{M}(x)\right\}$ a basis for the approximation of $u$ for a fixed $t$. Let

$$
\begin{gathered}
{[0, T]=\bigcup_{k}\left[t_{k}, t_{k+1}\right], \tau=t_{k+1}-t_{k} \text { and }} \\
a_{k}(t)=\frac{t_{k+1}-t}{\tau} \quad a_{k+1}(t)=\frac{t-t_{k}}{\tau} .
\end{gathered}
$$

The approximation of the minimizer of (11) is given by 


$$
\begin{aligned}
u_{h}(t, x)= & \sum_{j=1}^{M} \psi_{j}(x)\left(a_{k}(t) u_{j}^{k}+a_{k+1}(t) u_{j}^{k+1}\right) \\
& \text { for }(t, x) \in] t_{k}, t_{k+1}[\times \Omega .
\end{aligned}
$$

Theorem 1 There exists a sequence $u_{j}^{k+1}$ satisfying

$$
\begin{gathered}
\sum_{j=1}^{M} \mathcal{B}\left(\psi_{j} a_{k+1}, \psi_{i} a_{k+1}\right) \cdot u_{j}^{k+1}= \\
-\sum_{j=1}^{M} \mathcal{B}\left(\psi_{j} a_{k}, \psi_{i} a_{k+1}\right) \cdot u_{j}^{k}
\end{gathered}
$$

and converging toward the minimum of the function $J$.

The mathematical framework and a proof of this theorem is given in [10]. After integration of the relation (14) in time we obtain the least squares scheme with the finite element method approximating the minimizer of the functional $J$ defined by the relation (11):

$$
\begin{gathered}
\sum_{j=1}^{M} u_{j}^{k+1} \int_{\Omega}\left[\frac{\tau}{3}\left(\nabla \psi_{i} \mid v\right)\left(\nabla \psi_{j} \mid v\right)+\right. \\
\left(\frac{1}{2}+\frac{\tau}{3} \operatorname{div}(v)\right)\left(\left(\nabla \psi_{i} \mid v\right) \psi_{j}+\left(\nabla \psi_{j} \mid v\right) \psi_{i}\right)+ \\
\left.\left(\frac{1}{\tau}+\operatorname{div}(v)+\frac{\tau}{3}(\operatorname{div}(v))^{2}\right) \psi_{i} \psi_{j}\right] \mathrm{d} x= \\
-\sum_{j=1}^{M} u_{j}^{k} \int_{\Omega}\left[\frac{\tau}{6}\left(\nabla \psi_{i} \mid v\right)\left(\nabla \psi_{j} \mid v\right)+\right. \\
\left(-\frac{1}{2}+\frac{\tau}{6} \operatorname{div}(v)\right)\left(\nabla \psi_{i} \mid v\right) \psi_{j}+ \\
\left(\frac{1}{2}+\frac{\tau}{6} \operatorname{div}(v)\right)\left(\nabla \psi_{j} \mid v\right) \psi_{i}+ \\
\left.\left(-\frac{1}{\tau}+\frac{\tau}{6}(\operatorname{div}(v))^{2}\right) \psi_{i} \psi_{j}\right] \mathrm{d} x
\end{gathered}
$$

for all $i \in\{1, \cdots, M\}$. Note that for a velocity field with the null divergence $(\operatorname{div}(v)=0)$ the relation (15) corresponds to the STILS-time-marching scheme given in [1].

Figure 3 shows the segmentation result obtained with this scheme. We have considered a simple synthetic image representing a segment (see figure $3 a$ ). The pixels with high density correspond to the structure extracted from the image (see figure $3 \mathrm{e}$ ).

\section{Results}

We have tested the proposed method on confocal microscope images, described in section 1. In this study, segmentation was restricted to 2D. Figure 4 shows the segmentation results obtained with voting characteristics method proposed in the section 3.1. The center of

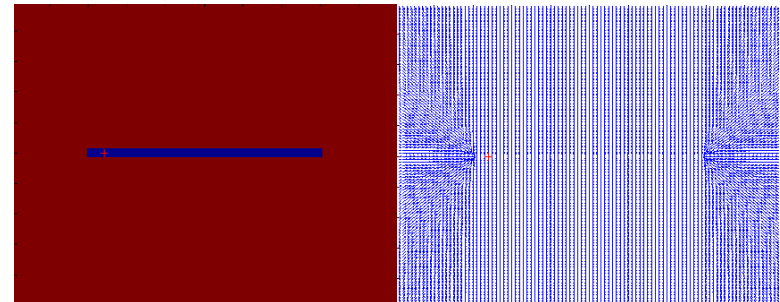

(a)

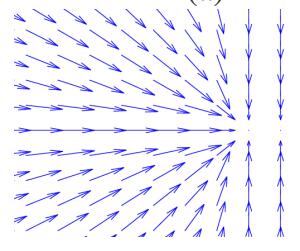

(d) (b)

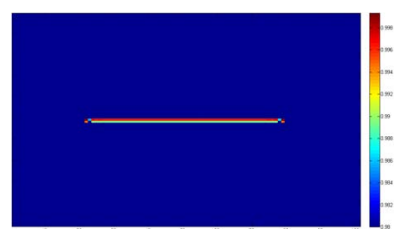

(e)
Figure 3. Voting by transport equation. (a) Synthetic image representing a segment; (b) velocity field computed from the image with the Fast Marching algorithm; (c) zoom on the velocity field shown in (b); (d) geodesic density computed by the relation (9) with the scheme (15).

the cell, which corresponds to the root of the propagation was chosen manually. The segmentation results are very satisfying. However, some parts of the tree were not present in the studied image, but could be found in the other 22 images of the image series. Hence, some segments of the tree extracted in the $2 \mathrm{D}$ segmentation do not correspond to a real contour. An extension of the proposed method to 3D segmentation should solve this problem.

\section{Discussion and conclusion}

In this work a method for the segmentation of tree structures was proposed that demanded the selection of just one point by the user. No a priori information about the tree structure was used, contrary to [4], where the length of the tree structure was given as a priori. The main contribution of this work is the introduction of geodesic density and its for tree stucture segmentation. To our knowledge this is the first time that geodesic voting is introduced. The numerical results obtained with geodesic voting are satisfying and encourage the extension to $3 \mathrm{D}$ segmentation. 


\section{Acknowledgements}

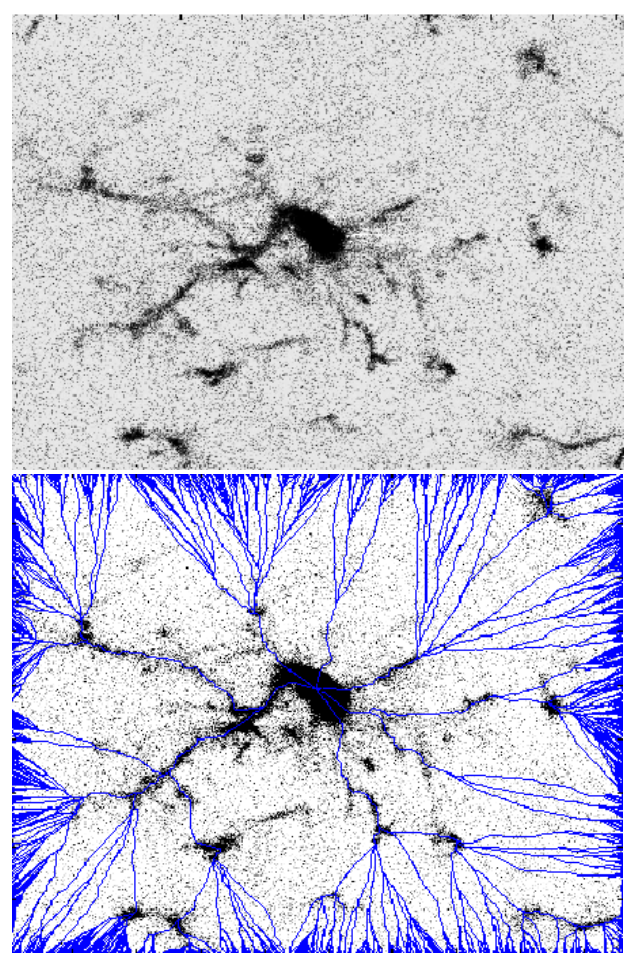

We are very grateful to A. Bessis and O. Pascual for providing the image sequences of microglia. We thank G. Carlier, G. Peyré, J. Pousin and F. Santambrogio for stimulating and useful discussions.

\section{References}

[1] O. Besson and G. de Montmollin. Space-time integrated least squares: a time-marching approach. Internat. $J$. Numer. Methods Fluids, 44(5):525-543, 2004.

[2] O. Besson and J. Pousin. Solutions for linear conservation laws with velocity fields in $L^{\infty}$. Arch. Ration. Mech. Anal., 186(1):159-175, 2007.

[3] L. Cohen. Minimal paths and fast marching methods for image analysis. In Handbook of mathematical models in computer vision, pages 97-111. Springer, New York, 2006.

[4] L. D. Cohen and T. Deschamps. Segmentation of 3D tubular objects with adaptive front propagation and minimal tree extraction for 3D medical imaging. Computer Methods in Biomechanics and Biomedical Engineering, 10(4):289-305, 2007.

[5] L. D. Cohen and R. Kimmel. Global minimum for active contour models: A minimal path approach. International Journal of Computer Vision, 24(1):57-78, 1997.

[6] M. G. Crandall and P.-L. Lions. Viscosity solutions of Hamilton-Jacobi equations. Trans. Amer. Math. Soc., 277(1):1-42, 1983.

[7] E. Fatemi, B. Engquist, and S. Osher. Numerical solution of the high frequency asymptotic expansion for the scalar wave equation. J. Comput. Phys., 120(1):145$155,1995$.

[8] R. Malladi and J. Sethian. Level set methods for curvature flow, image enchancement, and shape recovery in medical images, 1997.

[9] H. Nguyen and J. Reynen. A space-time least-square finite element scheme for advection-diffusion equations. Comput. Methods Appl. Mech. Eng., 42:331-342, 1984.

[10] Y. Rouchdy and L. Cohen. Image segmentation by geodesic voting. To be submitted, 2008.

[11] E. Rouy. Numerical approximation of viscosity solutions of first-order Hamilton-Jacobi equations with Neumann type boundary conditions. Math. Models Methods Appl. Sci., 2(3):357-374, 1992.

Figure 4. Results of the segmentation of microglia from confocal microscope images. Upper panel: microglia, middle panel: the geodesic paths (blue lines) are superposed on the image, lower panel: the extracted tree structure. The root corresponds to the cell center.

[12] J. Sethian. A fast marching level set method for monotonically advancing fronts. In Proc. Nat. Acad. Sci., volume 93, pages 1591-1595, 1996.

[13] J. A. Sethian. Level set methods and fast marching methods, volume 3 of Cambridge Monographs on Applied and Computational Mathematics. Cambridge University Press, Cambridge, second edition, 1999.

[14] J. Vidale. Finite-difference calculation of traveltime. $B$. Seismol. Soc. Am., 78:2062-2076, 1988. 